\title{
Mechanical stress promotes biological functions of C2C12 myoblasts by activating PI3K/AKT/mTOR signaling pathway
}

\author{
YU DA ${ }^{1}$, YONGBIN MOU ${ }^{2}$, MENGJIA WANG ${ }^{2}$, XIAO YUAN ${ }^{3}$, \\ FUHUA YAN $^{2}$, WEIDONG LAN ${ }^{2}$ and FANG ZHANG ${ }^{2}$
}

\begin{abstract}
${ }^{1}$ Department of Stomatology, Nanjing Tongren Hospital, School of Medicine, Southeast University, Nanjing, Jiangsu 211102;
${ }^{2}$ Department of Implantology, Nanjing Stomatological Hospital, Medical School of Nanjing University,

Nanjing, Jiangsu 210008; ${ }^{3}$ Department of Orthodontics, The Affiliated Qingdao Municipal Hospital,

Qingdao University, Qingdao, Shandong 266000, P.R. China
\end{abstract}

Received November 10, 2018; Accepted October 17, 2019

DOI: $10.3892 / \mathrm{mmr} .2019 .10808$

\begin{abstract}
The PI3K/AKT signaling pathway regulates cell proliferation and differentiation in multiple types of cells. The present study aimed to investigate the effects of mechanical stress on $\mathrm{C} 2 \mathrm{C} 12$ cell proliferation and to explore the associated mechanisms. A cyclic mechanical stress model of $\mathrm{C} 2 \mathrm{C} 12$ myoblasts was established. Reverse transcription-quantitative PCR and western blotting assay were used to examine the PI3K signaling pathways involved in the progress of cell differentiation. Cell counting kit-8 (CCK-8) assay was used to evaluate the proliferation of $\mathrm{C} 2 \mathrm{C} 12$ cells. Flow cytometry was employed to evaluate apoptosis following mechanical stress. The results demonstrated that mechanical stress activated the PI3K signaling pathway in $\mathrm{C} 2 \mathrm{C} 12$ myoblasts. Mechanical stress significantly promoted phosphorylation (p-) of AKT and expression of mammalian target of rapamycin (mTOR) compared with the normal group. Mechanical stress significantly promoted 4E-binding protein 1 (4EBP1) expression in $\mathrm{C} 2 \mathrm{C} 12$ cells compared with the normal group. The PI3K specific inhibitor LY294002 significantly decreased 4EBP1 expression and reduced p-AKT and p-mTOR expression compared with the mechanical stress group. Mechanical stress promoted $\mathrm{C} 2 \mathrm{C} 12$ cell proliferation. Apoptosis of $\mathrm{C} 2 \mathrm{C} 12$ significantly decreased in the mechanical stress group compared with the normal group. Cyclin D levels significantly increased in the mechanical stress group compared with the normal group. In conclusion, mechanical stress promoted biological functions of $\mathrm{C} 2 \mathrm{C} 12$ cells by activating the $\mathrm{PI} 3 \mathrm{~K} / \mathrm{AKT}$ signaling pathway. These results may contribute
\end{abstract}

Correspondence to: Dr Yongbin Mou, Department of Implantology, Nanjing Stomatological Hospital, Medical School of Nanjing University, 30 Zhongyang Road, Xuanwu, Nanjing, Jiangsu 210008, P.R. China

E-mail: mouyongbin@zoho.com.cn

Key words: PI3K/AKT signaling pathway, mechanical stress, $\mathrm{C} 2 \mathrm{C} 12$ cells, mammalian target of rapamycin to a better understanding of the effects of mechanical stress on cells.

\section{Introduction}

Myoblast stem cells are characterized by ability to self-renew and the potential to regenerate muscle. Myoblasts are mainly distributed in skeletal muscle tissues, but not in mature cardiac and smooth muscle tissues. Normally, myoblasts are present as muscle satellite cells. When muscles are damaged by external stimuli, myoblasts are activated and undergo proliferation and differentiation, finally becoming new muscle fibers. They therefore serve critical roles in muscle regeneration.

The PI3K/AKT signaling pathway participates in biological processes, including proliferation and differentiation. It is also involved in pathological processes of cancers and inflammations. PI3K activation phosphorylates and activates the plasma membrane located AKT molecule (1). By modulating expression of proteins, such as Bad, caspase-9 and runt-related transcription factor 2 , the PI3K/AKT signaling pathway inhibits cell apoptosis and triggers cell proliferation. The PI3K/AKT signaling pathway serves a significant role in the suppression of apoptosis and promotion of cell proliferation. AKT demonstrates a number of downregulatory effects by activating mTOR (2), which can affect transcription of p70 or 4E-binding protein 1 (4EBP1). mTOR, as a member of serine/threonine protein kinase family, tends to be affected by internal and external stimuli and can regulate the physiological activities of cells (2). In addition, the PI3K/AKT/mTOR signaling pathway is also considered to be an intracellular signaling pathway that can regulate cell cycles $(1,2)$.

mTOR can regulate cell proliferation, cell motility, protein synthesis, autophagy and transcription (3). The mTOR complex includes two sub-types, mTORc1 and mTORc2, both of which can be activated by molecules in the PI3K signaling pathway. mTORC1 activation is required for myofiber synthesis and skeletal muscle hypertrophy $(4,5)$. mTOR can transmit intracellular signals to downstream proteins and regulate cell proliferation. The most important downstream target of mTOR is p70S6K, which is a key molecule promoting protein synthesis and cell proliferation. PI3K/AKT/mTOR-S6K has 
been proven to be a classical signaling pathway in promoting cell differentiation and proliferation (3-5).

Mechanical stress can directly influence muscle growth by stretching myoblasts. In response to continuous force and injuries, myoblasts always shift to the influenced regions in order to repair the injuries. There are several molecules involving in mechanical stress, such as PI3K, NF- $\mathrm{BB}$ and p38MAPK, all of which are associated with cell differentiation, proliferation and apoptosis $(4,5)$. Overloading and continuous stress usually induces apoptosis of myoblasts through activating the $\mathrm{NF}-\kappa \mathrm{B}$ signaling pathway $(4,5)$. Therefore, it was hypothesized that there may be an association between mechanical stress loading-activated PI3K expression and cell proliferation. However, to the best of our knowledge, there are no studies investigating the roles of the PI3K/AKT/mTOR signaling pathway in the proliferation of myoblasts undergoing mechanical stress.

Therefore, the present study first investigated the effects of mechanical stress on the proliferation of myoblasts. Then, the signaling pathways that mediate stress-loading triggered proliferation of myoblast were explored by evaluating the expression of S6K, mTOR and AKT. Finally, the present study addressed whether mechanical stress involves the cell cycle by evaluating mTOR signaling pathway associated molecules.

\section{Materials and methods}

Cell culture and characterization of C2C12 myoblasts. C2C12 myoblasts were purchased from American Type Culture Collection. $\mathrm{C} 2 \mathrm{C} 12$ cells were warmed in a $37^{\circ} \mathrm{C}$ water bath and cultured in $5 \mathrm{ml}$ DMEM (Gibco; Thermo Fisher Scientific, Inc.) containing $10 \%$ fetal bovine serum (FBS; Gibco; Thermo Fisher Scientific, Inc.) at $37^{\circ} \mathrm{C}$. C2C12 cells were centrifuged at $1,000 \mathrm{x} \mathrm{g}$ for $5 \mathrm{~min}$ at $37^{\circ} \mathrm{C}$. The supernatant was removed and $1 \mathrm{ml}$ culture medium was added. Cells were diluted with medium containing $20 \% \mathrm{FBS}$ and cultured at $37^{\circ} \mathrm{C}$ with appropriate humidity and $5 \% \mathrm{CO}_{2}$ for6, $12,24,48$ or $72 \mathrm{~h}$, according to the different experimental protocols. In the indicated experiments, the $\mathrm{C} 2 \mathrm{C} 12$ cells were also treated with the PI3K inhibitor LY294002 (cat. no. A8250; APExBio Technology LLC).

Cells in culture bottles were washed with PBS2-3 times (10 min each time). Then, cells were digested using pancreatic enzyme (Beyotime Institute of Biotechnology) with shaking at $37^{\circ} \mathrm{C}$ for $2-5 \mathrm{~min}$. When cells had shrunk and fallen off the walls of the culture bottles, the cells were collected $(2-3 \mathrm{ml})$ and added to centrifugal tubes for following experiments or tests.

Establishment of cyclic mechanical stress model of C2C12 myoblasts. When adherent cells achieved a confluence of $70-80 \%$, they were washed with PBS2-3 times (10 min each time). The cells were then digested using pancreatic enzyme at $37^{\circ} \mathrm{C}$ for $2-5 \mathrm{~min}$. When cells had shrunk and fallen off the walls of the culture bottles, 2-3 ml of cells were collected, added to centrifuge tubes and adjusted to a density of $5 \times 10^{4}$ cells $/ \mathrm{ml}$. Then, the cells were added into 24-well plates (Corning, Inc.) for subsequent experiments. C2C12 myoblasts were administrated with cyclic mechanical stress at 5\% (stress 1 group), $10 \%$ (stress 2 group), $15 \%$ (stress 3 group) deformation and
$0.5 \mathrm{~Hz}$ (30 cycles/min) for 1, 6, 12 and $24 \mathrm{~h}$, using FX-4000 strain unit (Flexcell International Corp.). Cyclic mechanical stress loading was conducted at room temperature. Non-loaded control cells were cultured on a 24-well plate and kept in the same incubator. The mechanical stress loading experiment was conducted at least 3 times (10 min each time) and the data at each time-point were collected.

Reverse transcription-quantitative $(R T-q) P C R$. Total RNA of cultured cells was extracted using TRIzol ${ }^{\circledR}$ reagent (Invitrogen; Thermo Fisher Scientific, Inc.), according to manufacturer's instruction. cDNA was synthesized using the QuantiTect reverse transcription kit (cat. no. 205311; Qiagen $\mathrm{GmbH}$ ), following the supplier's protocol. The BeyoFast SYBR-Green qPCR Kit (cat. no. D7265; Beyotime Institute of Biotechnology) was used to amplify the target genes. The gene-specific primers used in the present study were synthesized based on the gene sequences listed in GenBank (https://www.ncbi.nlm.nih.gov/genbank/). Primer sequences for PCR are listed in Table I. The gene expression was quantified by normalizing to the GAPDH gene expression using the optimized comparative cycle threshold $\left(2^{-\Delta \Delta \mathrm{Cq}}\right)$ method (6).

Western blotting. Cells were digested and harvested in RIPA lysis buffer (Beyotime Institute of Biotechnology). The lysates were centrifuged at $15,000 \mathrm{xg}$ and $4^{\circ} \mathrm{C}$ for $30 \mathrm{sec}$. The concentrations of the lysates were evaluated using thebicinchoninic acid kit (cat. no. P0010S; Beyotime Institute of Biotechnology). Then, a total of $2 \mu \mathrm{g}$ proteins were separated using $8-15 \%$ SDS-PAGE, and electrotransferred onto polyvinylidene fluoride membranes (PVDF). The PVDF membranes were blocked with 5\% lipid milk in Tris-buffered saline containing $0.05 \%$ Tween-20 (TBST) for $2 \mathrm{~h}$ at room temperature. PVDF membranes were also incubated using primary antibodies $(1: 1,000)$ at $4^{\circ} \mathrm{C}$ overnight. Subsequently, PVDF membranes were incubated with secondary antibodies $(1: 1,000)$ at room temperature for $1.5 \mathrm{~h}$. Following washing with TBST four times (10 min each), protein signals were detected using an enhanced chemiluminescence kit (cat. no. 32109; Pierce; Thermo Fisher Scientific, Inc.). Primary antibodies were as follows: Rabbit anti-p-mTOR (cat. no. ab109268; Abcam), rabbit anti-p-AKT (cat. no. ab38448; Abcam), rabbit anti-4EBP1 (cat. no. ab32024; Abcam), rabbit internal anti- $\beta$-actin (cat. no. ab5694, Abcam). The secondary antibody sheep anti-rabbit IgG (cat. no. SAB3700918; Sigma-Aldrich; Merck KGaA) was used. When evaluating cyclin D expression, cells were separated into normal cells group and stress loading cells group. The primary antibodies, including rabbit anti-p-S6 (cat. no. ab109393; Abcam), rabbit anti-cyclin D (cat. no. ab16663; Abcam), rabbit internal GAPDH (cat. no. ab9485; Abcam) were used. The sheep anti-rabbit IgG (cat. no. SAB3700918; Sigma-Aldrich; Merck KGaA) was employed as the secondary antibody. Finally, the western blotting images were captured and analyzed using Labworks Analysis Software (version 3.0; Labworks LLC).

Cell counting kit-8 (CCK-8) assay. C2C12 cells were separated into normal group and normal + stress group, and cultured in $10 \%$ FBS solution. After inoculating in 96-well plates for $1-4 \mathrm{~h}, \mathrm{C} 2 \mathrm{C} 12$ cells were analyzed using the CCK-8 
Table I. Primer sequences for reverse transcription-quantitative PCR.

\begin{tabular}{lllll}
\hline Target gene & Gene ID & Primer & \multicolumn{1}{c}{ Primer sequence (5'-3') } & Fragment size (bp) \\
\hline PI3K & 18708 & Forward & AAGCCATTGAGAAGAAAGGACTG & 176 \\
& & Reverse & ATTTGGTAAGTCGGCGAGATAG & 121 \\
4EBP1 & \multirow{2}{*}{13685} & Forward & GGGAGGAACCAGGATTATCTATG & 263 \\
& & Reverse & ATCGCTGGTAGGGCTAGTGAC & \\
GAPDH & \multirow{2}{*}{14433} & Forward & GAGACCTTCAACACCCCAGC & \\
& & Reverse & ATGTCACGCACGATTCCC & \\
\hline
\end{tabular}

assay (cat. no. C0037; Beyotime Institute of Biotechnology), following the protocol of the manufacturer. The $450 \mathrm{~nm}$ absorbance was read using a microplate reader. A blank plate and control plate were also set up at the same time. Blank groups were treated with CCK-8 solution without cells, and control plate groups were treated with normal cells without stress administration. following the above tests, cell vitality was calculated and the cell inhibition rate evaluated.

Immunofluorescence assay. Cultured $\mathrm{C} 2 \mathrm{C} 12$ cells were washed using PBS three times (10 min each time), and then fixed with 4\% paraformaldehyde (Sigma-Aldrich; Merck $\mathrm{KGaA}$ ) for $15 \mathrm{~min}$ at room temperature. After treating cells with $0.5 \%$ Triton X-100 (Sigma-Aldrich; Merck KGaA) for $15 \mathrm{~min}, 6 \%$ goat serum (Gibco; Thermo Fisher Scientific, Inc.) was added for $30 \mathrm{~min}$ at room temperature. Then, cells were incubated with rabbit anti-PI3K monoclonal antibody (cat. no. ab32089; Abcam) overnight at $4^{\circ} \mathrm{C}$. After washing with PBS, cells were incubated with goat anti-rabbit Alexa Fluor 647-conjugated antibody (cat. no. ab150083;Abcam) for 30 min in the dark. Finally, cells were counted on a glass slide and observed using a confocal laser scanning microscope (FluoView FV1200; Olympus Corporation).

Flow cytometry assay. Cells at $60-70 \%$ confluence were harvested and treated with mechanical stress. Cells were washed twice with PBS (10 min each time) and centrifuged at 5,000 x $\mathrm{g}$ for $5 \mathrm{~min}$. Apoptosis was evaluated using the Annexin V-PE/7-AAD Apoptosis Detection Kit (cat. no. CA1030; Beijing Solarbio Science \& Technology Co., Ltd.). Briefly, $5 \mu 1$ 7-AAD dye solution in binding buffer was added to the cells at room temperature in the dark for 5-15 min, and then mixed with $450 \mu \mathrm{l}$ binding buffer. Following mixing with $1 \mu$ l Annexin V-PE for 5-15 min, cells were analyzed using a flow cytometer (Beckman Coulter, Inc.). The data was analyzed using the Flow Cytometer System II software (version 3.0; Beckman Coulter, Inc.).

Statistical analysis. Data were presented as mean \pm standard deviation and analyzed using GraphPad Prism 7 software (GraphPad Software, Inc.). Differences between treatment group and normal group were conducted using Student's t-test. Tukey's post-hoc test was used to validate the two-way analysis of variance for comparing differences among multiple groups. All of the experiments or tests were repeated $\geq 3$ times. $\mathrm{P}<0.05$ was considered to indicate a statistically significant difference.
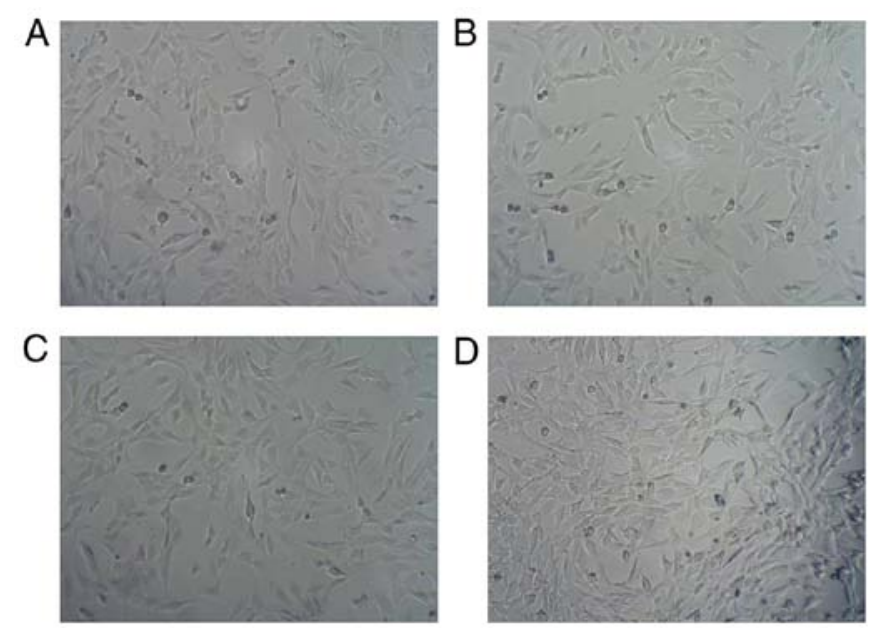

Figure 1. Cell morphology of $\mathrm{C} 2 \mathrm{C} 12$ cells. (A) $\mathrm{C} 2 \mathrm{C} 12$ cells after loading stress at $0 \mathrm{~h}$. (B) $\mathrm{C} 2 \mathrm{C} 12$ cells after loading stress at $6 \mathrm{~h}$. (C) $\mathrm{C} 2 \mathrm{C} 12$ cells after loading stress at $12 \mathrm{~h}$. (D) C2C12 cells after loading stress at $24 \mathrm{~h}$. Distribution of these cells conformed with stress. Magnification, $\mathrm{x} 400$.

\section{Results}

C2C12 myoblasts culture and characterization. Representative images of the features of $\mathrm{C} 2 \mathrm{C} 12$ cells are shown in Fig. 1. Regular cells were spread and distributed in Fig. 1A. After $6 \mathrm{~h}$ stress, cells were arranged regularly when compared with the normal group (Fig. 1B). Following 12 and $24 \mathrm{~h}$ cyclic stress, cells conforming to loading direction are shown in Fig. $1 \mathrm{C}$ and D.

Immunofluorescence. Due to the important roles of cycle stress in influencing the PI3K signaling pathway, immunofluorescence was used to observe the status of $\mathrm{C} 2 \mathrm{C} 12$ myoblasts. Cell counts of each group were compared with cell counts in the normal group (Fig. 2A). Cells after treatment with LY294002 and loading stress (stress 1), demonstrated obvious changes of morphology (Fig. 2). Comparing with the normal group, loading stress 1-treated cells were distributed following the tension direction and oriented in the direction of the stress source (Fig. 2A and B). Following the administration of inhibitors, the quantity of normal cells decreased (Fig. 2C). When cells were treated with cyclic tension and inhibitors together, cell numbers were slightly increased (Fig. 2D). Following exposure to increased stress (stress 2), the number of cells increased, and cell morphology changed to a shuttle shape (Fig. 2E). Nuclear fragmentation and lysates were barely 

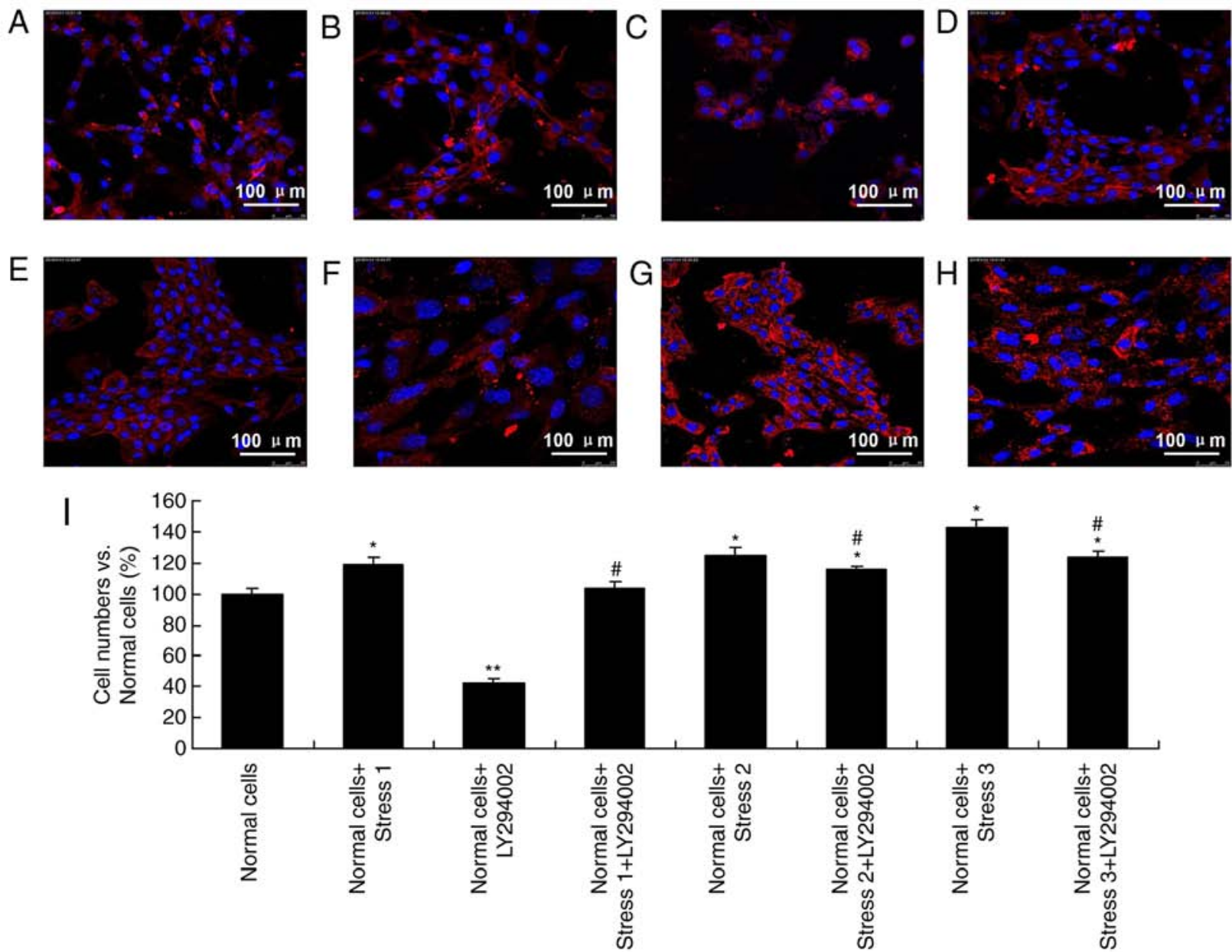

Figure 2. Immunofluorescence assay for $\mathrm{C} 2 \mathrm{C} 12$ cells. (A) Normal cells without stress. (B) Normal cells with stress 1 treatment. (C) Cells treated using inhibitor, LY294002 cells number decreased. (D) Cells underwent both stress 1 and LY294002 treatment; cell numbers increased, and cells were dispersed after undergoing mechanical stress 1. (E) After force strength loaded (stress 2) on normal cells, cell numbers increased, compared with (F) LY294002 treated cells. (G) Cells treated with the Stress 3. (H) Cells treated with the Stress 3 combining LY294002. (I) Statistical analysis for cell numbers vs. cell numbers in normal cells undergoing stress 3 treatment and/or LY294002. ${ }^{*} \mathrm{P}<0.05,{ }^{* *} \mathrm{P}<0.01$ vs. normal cells group; ${ }^{\#} \mathrm{P}<0.05$ vs. normal cells + Stress 1 , Stress 2 or Stress 3 group.

detected. Following inhibitor treatment, cells were scattered compared with cells in the normal group (Fig. 2E-G). In addition, following further stress (stress 3), the cells became apoptotic (Fig. 2G and H). A quantification of these changes is presented in Fig. 2I.

Mechanical stress upregulates PI3K in C2C12 cells. The mRNA expression levels of PI3K were determined using RT-qPCR. The results demonstrated that mechanical stress upregulated PI3K mRNA expression (Fig. 3). mRNA expression levels of PI3K decreased significantly following inhibitor treatment (Fig. 3). Following mechanical stress and inhibitor administration to $\mathrm{C} 2 \mathrm{C} 12$ cells, PI3K mRNA expression levels were clearly reduced compared with the single stress loading group (Fig. 3). These results suggest that the PI3K signaling pathway may be activated following mechanical stress loading.

Mechanical stress promotes phosphorylation of AKT and $m T O R$. To further examine whether the PI3K signaling pathway is activated by mechanical stress, western blotting was used to evaluate the phosphorylation of AKT and mTOR. First, $24 \mathrm{~h}$ loading stress was selected as the peak

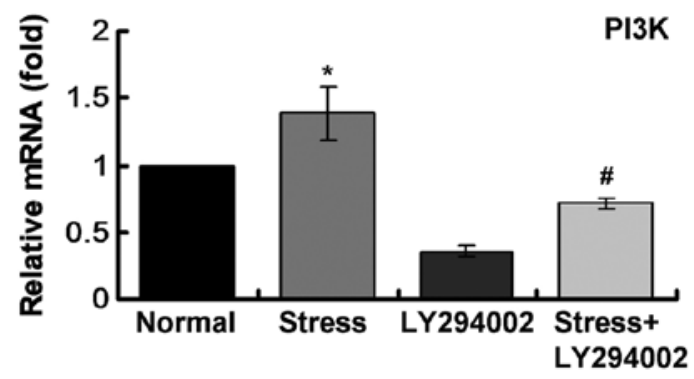

Figure 3. Mechanical stress upregulates PI3K. Stress loading on C2C12 increased mRNA expression levels of PI3K. Levels were decreased when both stress and LY294002 were administered to cells. ${ }^{*} \mathrm{P}<0.05$ vs. normal group; ${ }^{\#} \mathrm{P}<0.05$ vs. LY294002 group.

expression time-point and $\beta$-actin was used as the internal control. Following exposure to stress, p-AKT and p-mTOR reached their peak levels. Then, $\mathrm{C} 2 \mathrm{C} 12$ cells were treated with LY294002, and p-AKT/AKT and p-mTOR/mTOR ratios determined. The ratios of p-AKT/AKT and p-mTOR/mTOR declined significantly. With cells treated with both inhibitor and mechanical stress, expressions of p-mTOR and p-AKT significantly increased compared with inhibitor alone, as 


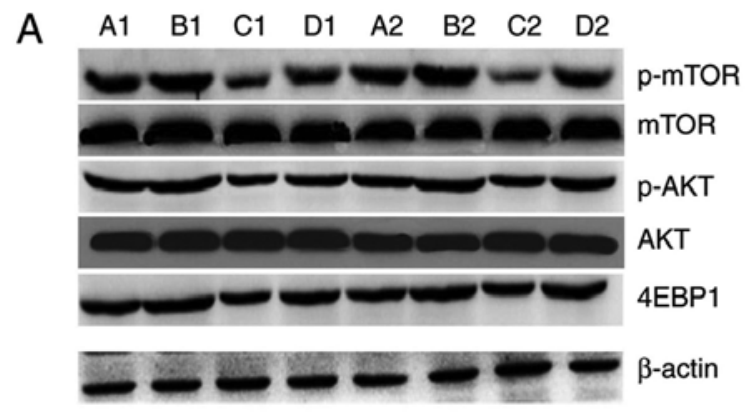

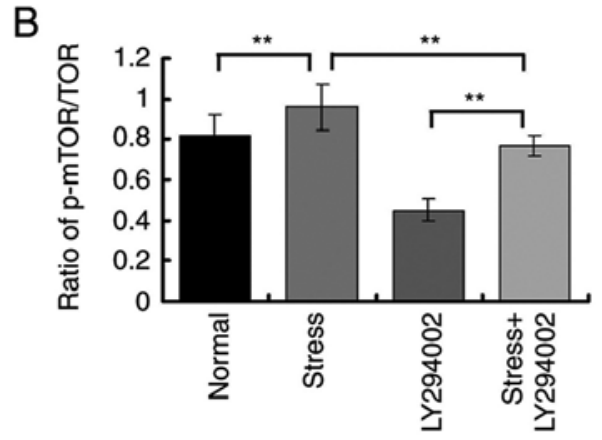
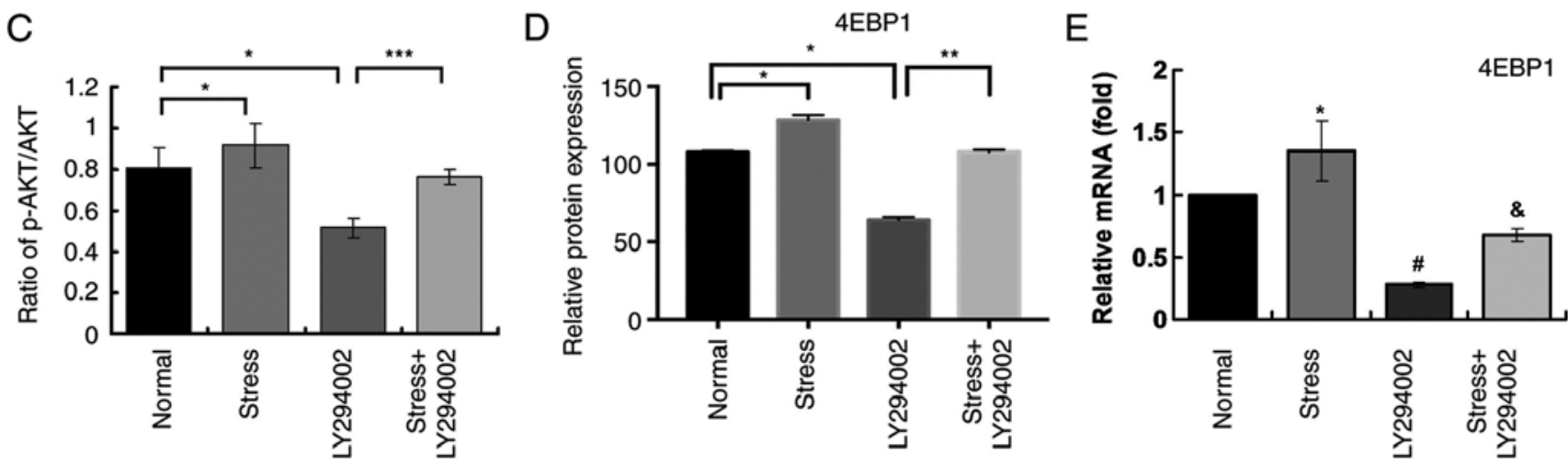

Figure 4. Effect of stress on $\mathrm{C} 2 \mathrm{C} 12$ cells through PI3K signaling pathway and 4EBP1 expression. (A) Western blot images for p-mTOR, mTOR, p-AKT, AKT and 4EBP1 in C2C12 cells. (B) Statistical analysis for ratio of p-mTOR/TOR. (C) Statistical analysis for ratio of p-AKT/AKT. (D) Statistical analysis for 4EBP1 expression. (E) Statistical analysis for 4EBP1 mRNA expression in $\mathrm{C} 2 \mathrm{C} 12$ cells. There were significant differences between normal cell group and stress group and between LY294002 exposure group and normal group. Values were presented as mean + standard deviation. A1 and A2 were assigned as normal cell group B1 and B2 were assigned as stress exposure group C1 and C2 were assigned as inhibitor LY294002 group D1 and D2 were assigned as LY294002+stress group. 4EBP1, 4E-binding protein 1; p-, phosphorylated. ${ }^{*} \mathrm{P}<0.05,{ }^{* *} \mathrm{P}<0.01,{ }^{* * *} \mathrm{P}<0.001$ vs. normal group; ${ }^{*} \mathrm{P}<0.05$ vs. Stress group; and ${ }^{\circledR} \mathrm{P}<0.05$ vs. LY294002 group.

demonstrated in Fig. 4A-C. It should be noted that ratios of p-AKT/AKT and p-mTOR/mTOR (phosphorylation of AKT and $\mathrm{mTOR}$ ) were lower in inhibitor-treated cells undergoing loading stress compared with the normal cells undergoing loading stress. Collectively, these results confirmed the hypothesis that cyclic stress not only activates PI3K signaling pathway but also increases the expression of mTOR.

Mechanical stress directly promotes 4EBP1 expression in C2C12 myoblasts by activating the PI3K pathway. As demonstrated in Fig. 4A and D, it was found that protein levels of 4EBPlincreased when stress loading was administrated to cells. Meanwhile, when blocking PI3K signaling pathway, 4EBP1 levels declined significantly. However, when treating cells with both inhibitor and stress, the protein expression levels of 4EBP1 were significantly reduced compared with the Stress alone group (Fig. 4A and D). Similar results were obtained for the mRNA expression levels of 4EBP1 by RT-qPCR analysis (Fig. 4E). Taken together, these results suggest that mechanical stress might modulate proliferation of myoblasts via activating the PI3K signaling pathway.

Mechanical stress promotes $\mathrm{C} 2 \mathrm{C} 12$ cell proliferation. $\mathrm{C} 2 \mathrm{C} 12$ cells undergoing stress loading were separated into the stress-loading group and the normal group. The stress-loading group demonstrated increased proliferation compared with the normal group, and this result was significant at $72 \mathrm{~h}$
$(\mathrm{P}<0.05$; Fig. 5). These data indicated that mechanical stress promoted $\mathrm{C} 2 \mathrm{C} 12$ proliferation.

Mechanical stress inhibits C2C12 cell apoptosis. To verify the effects of mechanical stress on cell apoptosis, flow cytometry assay was conducted. The results demonstrated that the apoptosis rate of the stress group was significantly lower compared with the normal group $(\mathrm{P}<0.05$; Fig. 6$)$. These results suggested that stress loading inhibited apoptosis in C2C12 cells.

Mechanical stress regulates the cell cycle by activating the PI3K/AKT/mTOR signaling pathway. An important cell cycle regulatory molecule, cyclin $\mathrm{D}$, was also detected. The results indicated that synthesis of cyclin D was initiated during G1 phase. Cyclin D expression increased after stress administration compared with the normal group ( $\mathrm{P}<0.05$; Fig. 7A and $\mathrm{B})$. As a key downstream protein of the mTOR signaling pathway, cyclin D can promote protein synthesis and trigger cell proliferation. Therefore, the results suggest that mechanical stress can modulate cell cycle and promote cell proliferation.

\section{Discussion}

A number of extracellular signaling pathways are involved in regulating myoblasts proliferation. Mechanical stress is considered to be a key factor in maintaining myoblast growth 


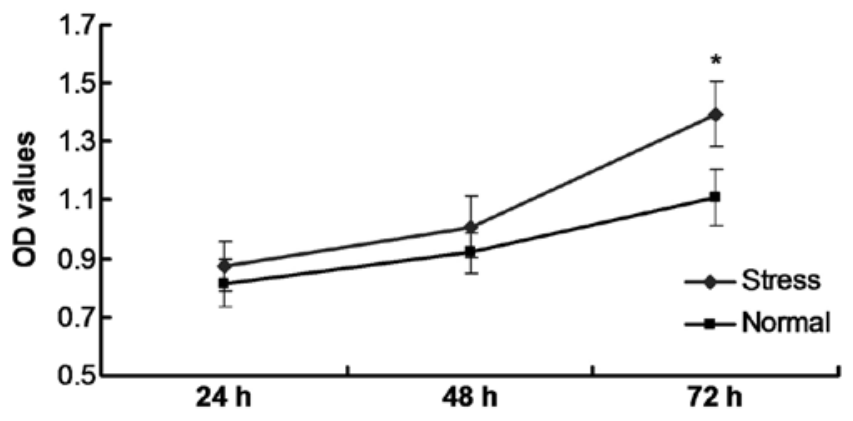

Figure 5. Cell proliferation was evaluated by Cell Counting Kit-8 assay. Compared with the normal cell group, proliferation in the stress cell group increased significantly at $72 \mathrm{~h}$. ${ }^{*} \mathrm{P}<0.05$ vs. normal group.

and survival. Mechanical stress can regulate metabolism and gene expression of myoblasts and serves important roles in skeletal muscle formation and development (7-10).

In order to explore biological characteristics of stressloading in myoblasts, intermittent cyclic stress was selected as an experimental strategy. Zhang et al (11) found that cyclic tensile stress serves critical roles in activating downstream signaling molecules, such as focal adhesion kinase (FAK) and Ras homolog gene family member A. Charrasse et al (12) identified that M-cadherin is an intermediate signaling pathway that is involved in the process of myoblasts fusing into muscle tubes. Kumar et al (13) demonstrated that cyclic stress can inhibit the differentiation of myoblasts by activating the FAK/Rac-1/GTPase/NF- $\kappa \mathrm{B}$ signaling pathway. Formigli et al (14) identified that ion-channels serve important roles in cyclic tensile processes. In addition, several other studies $(4,5,13)$ confirm that the $\mathrm{NF}-\kappa \mathrm{B}$ signaling pathway is involved in the apoptosis of myoblasts undergoing cyclic stress loading. Xiao et al (15) argued that stretch-induced connective tissue grow factor expression is mediated and modulated by the PI3K/JNK-dependent signaling pathway. However, few studies $(4,5,14,15)$ have clarified how the PI3K signaling pathway serves roles in the proliferation of myoblasts undergoing mechanical stress. In the present study, cyclic mechanical stress at $10 \%$ elongation with $0.5 \mathrm{~Hz}$ was used to simulate oral biomechanical stimulation on $\mathrm{C} 2 \mathrm{C} 12$ myoblasts.

The mRNA expression levels of several genes, including MyoD, Myogenin, MRF4 and Myf5, have been reported to be associated with mechanical stress (16). As a conclusive gene in differentiation of cells, MyoD promotes differentiation of myoblasts into skeletal muscles. MyoD and Myf5are usually over-expressed in myoblasts. In addition, recent researches $(13,15)$ have also demonstrated that $5 \%$ stretch stress can promote the proliferation of myoblasts. Stretch stress also inhibits Myogenin expression, which further regulates cell differentiation. When stretch is administrated at $>10 \%$, numbers of PARP shear content exist. Then, $15 \%$ stress with 10 cycles/min induces decreasing survival rates for myoblasts. Therefore, stress can accelerate proliferation, but higher stresses will promote apoptosis (17).

There are several factors involving in myoblasts proliferation, such as insulin-like growth factor 1 (IGF-1), zinc and follistatin. Mechanical stress constantly stimulates protein and mRNA synthesis in first hours of stretching (18), which is consistent with the results of the present study. Therefore, the relationship between mechanical stress and PI3K signaling pathway is precise. Many studies have demonstrated that stress activates proliferation, but over-expressed PI3K leads to tumor growth (13-15). PI3K/AKT is a classic pathway in connecting normal proliferating cells and tumor cells. The present study investigated the levels of p-AKT, p-mTOR and p-4EBP1. First, it was confirmed that stress can activate AKT. Second, levels of p-AKT decreased after the inhibition of the PI3K signaling pathway without stress. Third, it was also demonstrated that when both of stress and LY294002 were used on cells, levels of p-AKT only declined a little. These results suggest that mechanical stress may promote proliferation of myoblasts by activating the PI3K signaling pathway.

mTOR, an important downstream factor of the PI3K/AKT signaling pathway in transduction process, was also examined in the present study. Examination of mTOR may further demonstrate the cooperative effects of mechanical stress on the proliferation of cells via triggering PI3K signaling pathway. The present study demonstrated that mTOR also participates in mechanical stress induced cell proliferation. In addition, AKT promoted mTOR transcription by triggering mTOR mRNA expression in stress stimulated myoblasts. Blocking the PI3K signaling pathway resulted in a significant reduction of mTOR expression in cells undergoing stress loading. However, a number of other signaling pathways may also participate in this process.

Although expression of 4EBP1 was decreased, the levels were not stable. Therefore, unsteady expression of 4EBP1 was an unexpected finding. When two factors were administrated to myoblasts together, $\mathrm{p}-4 \mathrm{EBP} 1$ levels were unstable. This result suggested that PI3K/AKT had no direct effects on 4EBP1 proliferation, while higher expression of mTOR resulted in PI3K signaling pathway activation $(17,18)$. 4EBP1 expression is not influenced by mechanical stress directly, therefore there may be other mechanisms involving in 4EBP1 expression $(19,20)$.

The PI3K/AKT signaling pathway was activated by mechanical stress and resulted in increased p-mTOR expression, which further enhanced 4EBP1 expression. LY294002 treatment inhibited PI3K activation and reduced p-mTOR levels. In the present study, mRNA expression of PI3K signaling pathway associated genes was connected with p-mTOR expression, which suggested that activated mTOR can regulate targeting genes. Therefore, it can be concluded that the PI3K signaling pathway not only promoted cell proliferation, but also phosphorylated mTOR in mechanical stress loaded cells. mTOR serves an irreplaceable role in myoblasts proliferation under mechanical stress loading.

In order to confirm the proliferative effects of mechanical stress on $\mathrm{C} 2 \mathrm{C} 12$ cells, a CCK-8 assay was performed. The results demonstrated that $\mathrm{C} 2 \mathrm{C} 12$ proliferation was significantly increased following mechanical stress. It was hypothesized that proliferation and apoptosis may not always be consistently altered. Therefore, flow cytometry was conducted to examine the apoptosis rate. The results indicated that apoptosis was suppressed in mechanical stress-loaded cells. Therefore, stress inhibition may promote apoptosis, while enhanced mechanical stress in loading groups may result in increased proliferation by activating the PI3K signaling pathway. 

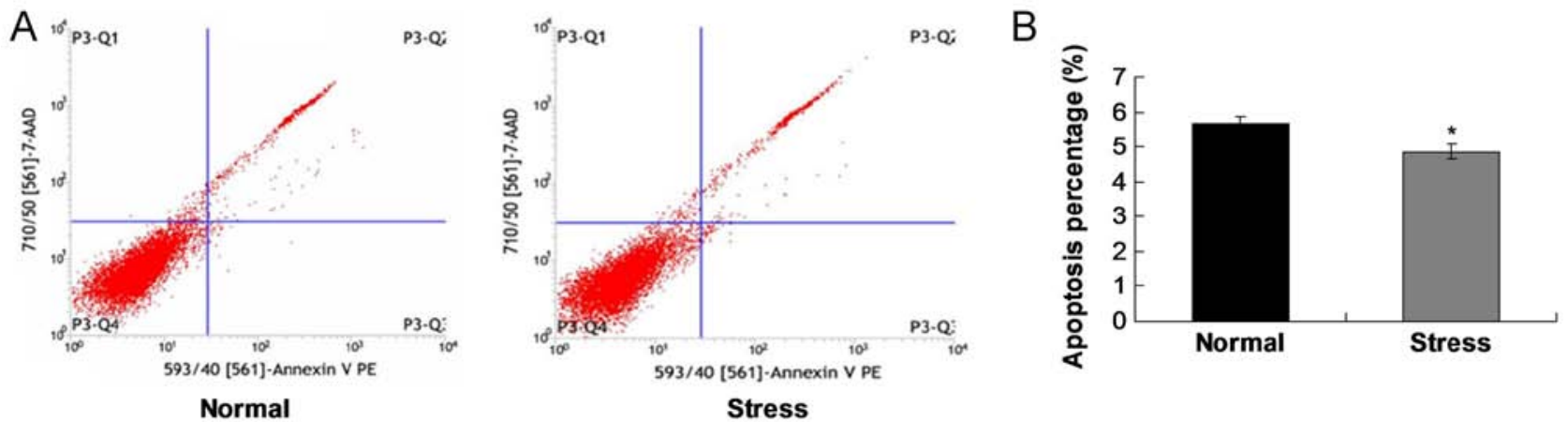

Figure 6. Evaluation for early apoptosis and late apoptosis. (A) Representative plots of flow cytometric analysis of apoptosis. (B) Statistical analysis of flow cytometric analysis. ${ }^{*} \mathrm{P}<0.05$ vs. normal group.

A

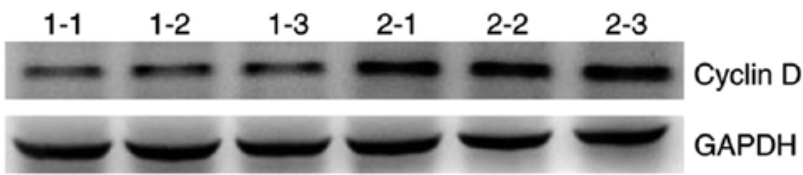

B

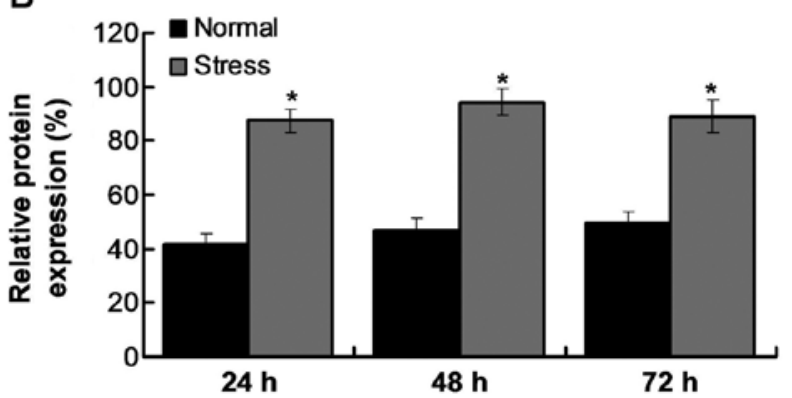

Figure 7. Evaluation for cyclin D expression using western blot assay. (A) Western blot analysis images. Lanes: 1-1, normal group $24 \mathrm{~h}$; 1-2, normal group $48 \mathrm{~h} ; 1-3$, normal group $72 \mathrm{~h} ; 2-1$, normal + stress group $24 \mathrm{~h} ; 2-2$, normal + stress group $48 \mathrm{~h}$; and 1-3 normal + stress group $72 \mathrm{~h}$. (B) Statistical analysis for cyclin $\mathrm{D}$ expression. ${ }^{*} \mathrm{P}<0.05$ vs. normal group at different time-points.

The effects of mechanical stress on $\mathrm{C} 2 \mathrm{C} 12$ proliferation may be associated with PI3K signaling pathway in two ways: i) PI3K significantly promotes cell proliferation of stress-stimulated C2C12 cells $(13,15)$, while other signaling pathways may also participate in the stress-mediated proliferation of cells; or ii) PI3K significantly inhibits stress-caused cell apoptosis (13-15). In the present study, when compared with normal cell groups, stress groups demonstrated a lower expression of PI3K.

A previous study reported that mTOR can be activated in distinct ways (21). mTOR can always be activated via phosphorylating or activating p70S6K in a mTOR-dependent pathway, triggering IGF-1/easing protein synthesis $(22,23)$. The present study also demonstrated that mechanical stress activated mTOR, especially for mTORc1.

Cyclin D mainly includes cyclin D1,cyclin D2 and cyclin D3 (24). Inhibition of cyclin D leads to cell cycle arrest and cell differentiation (25). Cyclin D is regulated by a downstream pathway of mitogen receptors via activating PI3K and glycogen synthase kinase three $\beta$ (GSK3 $\beta$ ) (25). GSK3 $\beta$ can also cause cyclin $\mathrm{D}$ degradation by inhibiting phosphorylation of cyclin D molecules (25). GSK3 $\beta$ is negatively modulated by $\mathrm{PI} 3 \mathrm{~K}$ signaling pathway in form of phosphorylation, and is associated with the expression of cyclin D (25). The present study established cyclin D as a biomarker for C2C12 cells undergoing stress stimuli. Its results suggested that mechanical stress can promote cell cycle of $\mathrm{C} 2 \mathrm{C} 12$ cells by activating proliferation and activating the PI3K signaling pathway. However, the deeper mechanisms of this process have not been elucidated in the present study.

A few published studies (26-28) report that proliferation of $\mathrm{C} 2 \mathrm{C} 12$ myoblasts is associated with the PI3K/AKT/mTOR signaling pathway. However, the present study investigated the effects of mechanical stress on $\mathrm{C} 2 \mathrm{C} 12$ proliferation by exploring the PI3K/AKT signaling pathway and evaluating the apoptosis process for the first time, to the best of the authors' knowledge. The present study provided insight for clarifying the molecular mechanism of intracellular stress in C2C12 cells and may be of benefit to studies investigating skeletal muscle cell associated disorders.

Although the present study provided several notable results, there were also a few limitations. First, it only used CCK-8 assay to evaluate proliferation of $\mathrm{C} 2 \mathrm{C} 12$ cells. It might be more convincing for the conclusions if other methods had been used to examine proliferation. Second, the specific mechanism for the potential effects of stress loading on cell proliferation has not been clarified. Future studies should examine the cell cycle phase distribution of cells undergoing stress stimuli. Third, the expression levels of the total and the phosphorylated PI3K were not examined in the present study.

In conclusion, the present study demonstrated that mechanical stress promotes $\mathrm{C} 2 \mathrm{C} 12$ proliferation by activating the PI3K/AKT signaling pathway and inhibiting the apoptosis process. These results would contribute to a better understanding for mechanism of functional appliance via PI3K/AKT signaling pathway.

\section{Acknowledgements}

Not applicable.

\section{Funding}

The present study was supported by The Nanjing Science and Technology Development Project (2016; grant no. 201605067). 


\section{Availability of data and materials}

All data generated or analyzed during this study are included in this published article.

\section{Authors' contributions}

YD, YM, MW and XY performed the experiments. YD and YM designed the study and wrote the manuscript. FY and FZ conducted the statistical analysis. WL conducted the literature review. All authors read and approved the final manuscript.

\section{Ethics approval and consent to participate}

Not applicable.

\section{Patient consent for publication}

Not applicable.

\section{Competing interests}

The authors declare that they have no competing interests.

\section{References}

1. Zhang B, Liu Y, Li Y, Zhe X, Zhang S and Zhang L: Neuroglobin promotes the proliferation and suppresses the apoptosis of glioma cells by activating the PI3K/AKT pathway. Mol Med Rep 17: 2757-2763, 2018.

2. Rafalski VA and Brunet A: Energy metabolism in adult neural stem cell fate. Prog Neurobiol 93: 182-203, 2011.

3. Hay N and Sonenberg N: Upstream and downstream of mTOR. Genes Dev 18: 1926-1945, 2004.

4. Brook MS, Wilkinson DJ, Phillips BE, Perez-Schindler J, Philp A, Smith K and Atherton PJ: Skeletal muscle homeostasis and plasticity in youth and ageing: Impact of nutrition and exercise. Acta Physiol (Oxf) 216: 15-41, 2016.

5. Brioche T, Pagano AF, Py G and Chopard A: Muscle wasting and aging: Experimental models, fatty infiltrations, and prevention. Mol Aspects Med 50: 56-87, 2016.

6. Livak KJ and Schmittgen TD: Analysis of relative gene expression data using real-time quantitative PCR and the 2 (-Delta Delta C(T)) method. Methods 25: 402-408, 2001.

7. Fujita H, Hida M, Kanemoto K, Fukuda K, Nagata $M$ and Awazu M: Cyclic stretch induces proliferation and TGF-beta1-mediated apoptosis via p38 and ERK in ureteric bud cells. Am J Physiol Renal Physiol 299: F648-F655, 2010.

8. Nakai N, Kawano F, Oke Y, Nomura S, Ohira T, Fujita R and Ohira Y: Mechanical stretch activates signaling events for protein translation initiation and elongation in $\mathrm{C} 2 \mathrm{C} 12$ myoblasts. Mol Cells 30: 513-518, 2010.

9. Wang BW, Chang H and Shyu KG: Regulation of resistin by cyclic mechanical stretch in cultured rat vascular smooth muscle cells. Clin Sci (Lond) 118: 221-230, 2009.

10. Shyu KG, Wang BW, Lin CM and Chang H: Cyclic stretch enhances the expression of toll-like receptor 4 gene in cultured cardiomyocytes via p38 MAP kinase and NF-kappaB pathway. J Biomed Sci 17: 15, 2010.

11. Zhang SJ, Truskey GA and Kraus WE: Effect of cyclic stretch on beta1D-integrin expression and activation of FAK and RhoA. Am J Physiol Cell Physiol 292: C2057-C2069, 2007.
12. Charrasse S, Comunale F, Fortier M, Portales-Casamar E, Debant A and Gauthier-Rouviere C: M-cadherin activates Rac1 GTPasethrough the Rho-GEF trio during myoblast fusion. Mol Biol Cell 18: 1734-1743, 2007.

13. Kumar A, Murphy R, Robinson P, Wei L and Boriek AM: Cyclic mechanical strain inhibits skeletal myogenesis through activation of focal adhesion kinase, Rac-1 GTPase, and NF-kappaB transcription factor. FASEB J 18: 1524-1535, 2004.

14. Formigli L, Meacci E, Sassoli C, Squecco R, Nosi D, Chellini F, Naro F, Francini F and Zecchi-Orlandini S: Cytoskeleton/ stretch-activated ion channel interaction regulates myogenic differentiation of skeletal myoblasts. J Cell Physiol 211: 296-306, 2007.

15. Xiao LW, Yang M, Dong J, Xie H, Sui GL, He YL, Lei JX, Liao EY and Yuan X: Stretch-inducible expression of connective tissue growth factor (CTGF) in human osteoblasts-like cells is mediated by PI3K-JNK pathway. Cell Physiol Biochem 28: 297-304, 2011.

16. Abe S, Rhee S, Iwanuma O, Hiroki E, Yanagisawa N, Sakiyama K and Ide Y: Effect of mechanical stretching on expressions of muscle specific transcription factors MyoD, Myf-5, myogenin and MRF4 in proliferated myoblasts. Anat Histol Embryol 38: 305-310, 2009.

17. Peltier J, O'Neill A and Schaffer DV: PI3K/Akt and CREB regulate adult neural hippocampal progenitor proliferation and differentiation. Dev Neurobiol 67: 1348-1361, 2007.

18. Sui L, Wang J and Li BM: Role of the phosphoinositide 3-kinase-Akt-mammalian target of the rapamycin signaling pathway in long-term potentiation and trace fear conditioning memory in rat medial prefrontal cortex. Learn Mem 15: 762-776, 2008.

19. Datan E, Shirazian A, Benjamin S, Matassov D, Tinari A, Malorni W, Lockshin RA, Garcia-Sastre A and Zakeri Z: mTOR/p70S6K signaling distinguishes routine, maintenance-level autophagy from autophagic cell death during influenza A infection. Virology 452-453: 175-190, 2014.

20. Ci Y, Shi K, An J, Yang Y, Hui K, Wu P, Shi L and Xu C: ROS inhibit autophagy by downregulating ULK1 mediated by the phosphorylation of p53 in selenite-treated NB4 cells. Cell Death Dis 5: e1542, 2014

21. Chung J, Kuo CJ, Crabtree GR and Blenis J: Rapamycin-FKBP specifically blocks growth-dependent activation of and signaling by the 70 kd S6 protein kinases. Cell 69: 1227-1236, 1992.

22. Chiang GG and Abraham RT: Phosphorylation of mammalian target of rapamycin (mTOR) at Ser-2448 is mediated by p70S6 kinase. J Biol Chem 280: 25485-25490, 2005.

23. Rahman H, Qasim M, Oellerich M and Asif AR: Identification of the novel interacting partners of the mammalian target of rapamycin complex 1 in human CCRF-CEM and HEK293 cells. Int J Mol Sci 15: 4823-4836, 2014.

24. Chang H, Liu YH, Wang LL, Wang J, Zhao ZH, Qu JF and Wang SF: MiR-182 promotes cell proliferation by suppressing FBXW7 and FBXW11 in non-small cell lung cancer. Am J Transl Res 10: 1131-1142, 2018.

25. Diehl JA, Cheng M, Roussel MF and Sherr CJ: Glycogen synthase kinase-3beta regulates cyclin D1 proteolysis and subcellular localization. Genes Dev 12: 3499-3511, 1998.

26. Matheny RW and Adamo ML: Effects of PI3K catalytic subunit and Akt isoform deficiency on mTOR and p70S6K activation in myoblasts. Biochem Biophys Res Commum 390: 252-257, 2009.

27. Hu SY, Tai CC, Li YH and WU JL: Progranulin compensates for blocked IGF-1 signaling to promote myotube hypertrophy in C2C12 myoblasts via the PI3K/Akt/mTOR pathway. FEBS Lett 586: 3485-3492, 2012.

28. Kitakaze T, Sakamoto T, Kitano T, Inoue N, Sugihara F, Harada N and Yamaji R: The collagen derived dipeptide hydroxyprolyl-glycine promotes $\mathrm{C} 2 \mathrm{C} 12$ myoblast differentiation and myotube hypertrophy. Biochem Biophys Res Commun 478: 1292-1297, 2016. 